International Journal of Pure and Applied Mathematics

Volume 96 No. 1 2014, 105-115

ISSN: 1311-8080 (printed version); ISSN: 1314-3395 (on-line version)

url: http://www.ijpam.eu

doi: http://dx.doi.org/10.12732/ijpam.v96i1.8

ijpam.eu

\title{
ON THE STRATIFICATION OF THE PROJECTIVE SPACE BY THE $X$-RANK FOR A CERTAIN CONFIGURATION $X$ OF RATIONAL NORMAL CURVES
}

\author{
E. Ballico \\ Department of Mathematics \\ University of Trento \\ 38123 Povo (Trento) - Via Sommarive, 14, ITALY
}

\begin{abstract}
Let $\nu_{d}: \mathbb{P}^{m} \rightarrow \mathbb{P}^{N}, m \geq 2, N:=\left(\begin{array}{c}m+d \\ m\end{array}\right)-1$, denote the Veronese embedding. Fix $O \in \mathbb{P}^{m}$ and let $T_{m}$ be the union of $m$ lines of $\mathbb{P}^{m}$ passing through $O$ and with $\left\langle T_{m}\right\rangle=\mathbb{P}^{m}$, where \langle\rangle denote the linear span $\left(T_{m}\right.$ is an angle or a coordinate frame). Set $T_{m, d}:=\nu_{d}\left(T_{m}\right)$. In this note we study the stratification by $T_{m, d}$-rank of $\left\langle T_{m, d}\right\rangle$. We give a sharp upper bound for this rank, prove a concision result (in a precise quantitative way) and study some cases with high rank.
\end{abstract}

AMS Subject Classification: 14N05

Key Words: symmetric tensor rank, reducible curve, border rank, rational normal curve

\section{Introduction}

For all positive integers $m, d$ let $\nu_{d}: \mathbb{P}^{m} \rightarrow \mathbb{P}^{N}, N:=\left(\begin{array}{c}m+d \\ m\end{array}\right)-1$, denote the Veronese embedding induced by $\left|\mathcal{O}_{\mathbb{P} m}(d)\right|$. Set $X_{m, d}:=\nu_{d}\left(\mathbb{P}^{m}\right)$.

Fix an integer $m \geq 2, O \in \mathbb{P}^{m}$ and $m$ lines $L_{1}, \ldots, L_{m} \subset \mathbb{P}^{m}$ such that $O \in L_{i}$ for all $i$ and $\left\langle L_{1} \cup \cdots \cup L_{m}\right\rangle=\mathbb{P}^{m}$, where \langle\rangle denote the linear span. Set 
$T_{m}:=L_{1} \cup \cdots \cup L_{m}$ and $T_{m, d}:=\nu_{d}\left(T_{m}\right)$. The curves $T_{m}$ and $T_{m, d}$ do not depend upon the choice of $O$ and the lines $L_{1}, \ldots, L_{m}$, up to a projective equivalence. The curve $T_{m}$ is reduced, connected, $p_{a}\left(T_{m}\right)=0$ and its unique singular point, $O$, is a seminormal singularity with multiplicity $m$ ( $T_{2}$ is a reducible conic). We will say that $T_{m}$ is an angle or a coordinate frame. The curve $\nu_{d}\left(T_{m}\right)$ has degree $d m$, each $\nu_{d}\left(L_{i}\right)$ is a degree $d$ rational normal curve in its linear span $\left\langle\nu_{d}\left(L_{i}\right)\right.$ and $\operatorname{dim}\left(\left\langle T_{m, d}\right\rangle\right)=m d$. For each $P \in\left\langle T_{m, d}\right\rangle$, its $T_{m, d} \operatorname{rank} r_{T_{m, d}}(P)$ is the minimal cardinality of a set $S \subset T_{m}$ such that $P \in\left\langle\nu_{d}(S)\right\rangle$. For each $P \in \mathbb{P}^{N}$ the symmetric tensor rank $r_{m, d}(P)$ of $P$ is the minimal cardinality of a subset $S \subset \mathbb{P}^{m}$ such that $P \in\left\langle\nu_{d}(S)\right\rangle$. If $P \in\left\langle T_{m, d}\right\rangle$, then both $r_{m, d}(P)$ and $r_{T_{m, d}}(P)$ and obviously $r_{m, d}(P) \leq r_{T_{m, d}}(P)$. In the (omitted) case $m=1$ concision says that equality holds ([7]) In the case $m \geq 2$ easy examples shows that often strict inequality holds (Example 2), but in some interesting cases equality holds (Proposition 4).

Let $2 O$ be the closed subscheme of $\mathbb{P}^{m}$ with $\left.\left(\mathcal{I}_{O}\right)^{2}\right)$ as its ideal sheaf. We have $2 O \subset T_{m}$ and $\left\langle\nu_{d}(2 O)\right\rangle$ is an $m$-dimensional linear subspace of $\mathbb{P}^{N}$ which is the tangent space of the Veronese variety $X_{m, d}$ at $O$. For each closed subscheme $U \subseteq \mathbb{P}^{m}$ set $\{2 O, U\}:=2 O \cap U$.

Remark 1. Fix $P \in\left\langle T_{m, d}\right\rangle$ such that $P \notin\left\langle\nu_{d}(T)\right\rangle$ for any proper subcurve $T$ of $T_{m}$. There are $P_{i} \in\left\langle\nu_{d}\left(L_{i}\right)\right\rangle \backslash\left\{\nu_{d}(O)\right\rangle$ such that $P \in\left\langle\left\{P_{1}, \ldots, P_{n}\right\}\right\rangle$. The points $P_{1}, \ldots, P_{n}$ are not unique, but if $Q_{1}, \ldots, Q_{n}$ are another choice, then $Q_{i} \in\left\langle\left\{\nu_{d}(O), P_{i}\right\}\right\rangle$. Hence $P_{i}$ is contained in the tangent line $\left\langle\nu_{d}\left(2 O, L_{i}\right\}\right\rangle$ of $\nu_{d}\left(L_{i}\right)$ if and only if $Q_{i}$ is contained in the tangent line $\left\langle\nu_{d}\left(2 O, L_{i}\right\}\right\rangle$ of $\nu_{d}\left(L_{i}\right)$. Hence the number $\rho(P)$ of such points $P_{i}$ depends only from $P$. Now take any $P \in\left\langle T_{m, d}\right\rangle \backslash\left\{\nu_{d}(O)\right\}$ and call $T$ a minimal subcurve of $T_{m}$ such that $P \in\langle\nu(T)\rangle$. If $T \neq T_{m}$, then define $\rho(P)$ using this curve $T \cong T_{x}, 1 \leq x<m$. In particular, if $\rho(P)=m$, then $P \notin\left\langle\nu_{d}(T)\right\rangle$ for any proper subcurve $T$ of $T_{m}$.

Theorem 1. Assume $d \geq 3$. For any $P \in\left\langle T_{m, d}\right\rangle$ we have $r_{T_{m, d}}(P) \leq$ $m(d-1)+1+\min \{n-1, \rho(P)-1\}$. If $d \geq 5$, then $r_{T_{m, d}}(P)=$ md if and only if $\rho(P)=m$.

For points $P \in\left\langle\nu_{d}(2 O)\right\rangle$, see Proposition 2.

We also consider a scheme $Z \subset T_{m}$ with $Z \supset 2 O$ and $\operatorname{deg}(Z)=\operatorname{dim}(2 O)+1$, give an upper bound for a point $P \in\left\langle\nu_{d}(Z)\right\rangle \backslash\left\langle\nu_{d}(2 O)\right\rangle$ (Proposition 3) and prove that if $m=3$, then this upper bound $3 d-3$ is the exact value both of $r_{T_{3, d}}(P)$ and $r_{3, d}(P)$ (Proposition 4). A kind of concision with very precise bounds holds for a proper subscurve $T \subsetneq T_{m}$ : if $P \in\left\langle\nu_{d}(T)\right\rangle$ and $S \subset T_{m}$ is a finite subset such that $P \in\left\langle\nu_{d}(S)\right\rangle \backslash\left\langle\nu_{d}(S \cap T)\right\rangle$, then $\sharp(S) \geq r_{T_{m, d}}(P)+d$ (Proposition 1). Easy examples shows that sometimes this inequality is sharp 
(Example 1).

\section{The proofs}

Linear algebra (or Mayer-Vietoris exact sequences obtained from decompositions like $\left.T_{m}=T_{m-1} \cup L_{m}\right)$ gives the following lemma.

Lemma 1. Fix $I, J \subseteq\{1, \ldots, m\}$ such that $I \neq \emptyset$ and $J \neq \emptyset$. If $I \cap J=\emptyset$, then $\left\langle\nu_{d}\left(\cup_{i \in I} L_{i}\right)\right\rangle \cap\left\langle\nu_{d}\left(\cup_{i \in J} L_{i}\right)\right\rangle=\nu_{d}(O)$. If $I \cap J \neq \emptyset$, then $\left\langle\nu_{d}\left(\cup_{i \in I} L_{i}\right)\right\rangle \cap$ $\left\langle\nu_{d}\left(\cup_{i \in J} L_{i}\right)\right\rangle=\left\langle\nu_{d}\left(\cup_{i \in I \cap J} L_{i}\right)\right\rangle$.

Lemma 2. Fix any $S \subset T_{m}$ such that $\nu_{d}(O) \in\left\langle\nu_{d}(S)\right\rangle$ and $O \notin S$. Then $\sharp(S) \geq d+1$ and if $\sharp(S) \leq 2 d$, then there is a line $L \subset T_{m}$ such that $\sharp(S \cap L) \geq d+1$.

Proof. Taking if necessary a smaller $S$ we may assume that $\nu_{d}(S)$ is linearly independent and in particular that no $d+2$ of the points of $S$ are collinear. Since $O \notin S,[4$, Lemma 34] gives $\sharp(S \cup\{O\}) \geq d+2$ and that if $\sharp(S \cup\{O\}) \leq 2 d+1$, then there is a line $L \subset \mathbb{P}^{m}$ such that $\sharp((S \cup\{O\}) \cap L) \geq d+2$. Since $\sharp(S \cap L) \geq 3$ and $T_{m}$ is cut out by quadrics, $L=L_{i}$ for some $i$.

The first part of the next result is related to the concision property for multivariate polynomials ([7, Exercise 3.2.2.2]).

Proposition 1. Fix $P \in\left\langle T_{m, d}\right\rangle$ and assume the existence of a curve $T \subsetneq T_{m}$ such that $P \in\left\langle\nu_{d}(T)\right\rangle$. Then:

1. each subset of $T_{m}$ evincing $r_{T_{m, d}}(P)$ is contained in $T$, i.e. $S \subset T$ for each $S \subset T_{m}$ such that $\sharp(S)=r_{T_{m, d}}(P)$ and $P \in\left\langle\nu_{d}(S)\right\rangle$;

2. take any finite $S \subset T_{m}$ such that $P \in\left\langle\nu_{d}(S)\right\rangle$ and $P \notin\left\langle\nu_{d}(S \cap T)\right\rangle$. Then $\sharp(S)-\sharp(S \cap T) \geq d+1$ and $\sharp(S) \geq r_{T_{m, d}}(P)+d$.

Proof. It is sufficient to prove part (2). Take any $S \subset T_{m}$ such that $P \in\left\langle\nu_{d}(S)\right\rangle$ and $P \notin\left\langle\nu_{d}(S \cap T)\right\rangle$. Write $T_{m}=T \cup T^{\prime}$ with $T^{\prime}$ the union of the lines of $T_{m}$ not contained in $T$. We have $T \cap T^{\prime}=\{O\}$. We have $P \in\left\langle\left\langle\nu_{d}\left(S \cap T^{\prime}\right)\right\rangle \cup\left\langle\nu_{d}(T \cap S)\right\rangle\right\rangle$. Since $\left\langle\nu_{d}\left(T^{\prime}\right)\right\rangle \cap\left\langle\nu_{d}(T)\right\rangle=\left\{\nu_{d}(O)\right\}$ and $P \notin\left\langle\nu_{d}(S \cap T)\right\rangle$, we get $O \notin S, P \in\langle\{O\} \cup(S \cap T)\rangle$ and $O \in\left\langle\nu_{d}(T \cap S)\right\rangle$. Hence it is sufficient to prove that $\sharp\left(T^{\prime} \cap S\right) \geq d+1$. Apply Lemma 2 .

Example 1. The following example shows that part (2) of Proposition 1 is sharp. Take any $P \in\left\langle T_{m-1, d}\right\rangle$ and any $S \subset T_{m}$ such that $P \in\left\langle\nu_{d}(S)\right\rangle$ 
and $P \notin\left\langle\nu_{d}\left(S \cap T_{m-1}\right)\right\rangle$. Since $P \in\left\langle\left\langle\left(\nu_{d}\left(S \cap L_{m}\right)\right\rangle \cup\left\langle\nu_{d}\left(S \cap T_{m-1}\right)\right\rangle, P \notin\right.\right.$ $\left\langle\nu_{d}\left(S \cap T_{m-1}\right)\right\rangle$ and $\left\langle\nu_{d}\left(L_{m}\right)\right\rangle \cap\left\langle\nu_{d}\left(T_{m-1}\right)\right\rangle=\{O\}$ (Lemma 1), we get $O \notin S$, $P \in\left\langle\nu_{d}\left(\{O\} \cup\left(S \cap T_{n-1}\right)\right\rangle\right.$ and $O \in\left\langle\nu_{d}\left(L_{n} \cap S\right)\right\rangle$. Since $O \notin L_{n} \cap S$, we get $\sharp\left(L_{n} \cap S\right) \geq d+1$. Fix any $A \subset L_{n}$ such that $O \notin A$ and $\sharp(B)=d+1$. For any $B \subset T_{n-1}$ such that $O \in B, P \in\left\langle\nu_{d}(B)\right\rangle$ and $P \notin\left\langle\nu_{d}\left(B^{\prime}\right)\right\rangle$ for any $B^{\prime} \subsetneq B$. Set $E:=A \cup(B \backslash\{O\})$. We have $P \in\left\langle\nu_{d}(E)\right\rangle$ and $\sharp(E)=d+\sharp(B)$. Part (2) of Proposition 1 and Lemma 1 give $\left.P \notin \nu_{d}\left(E^{\prime}\right)\right\rangle$ for any $E^{\prime} \subsetneq E$.

Proposition 2. Assume $d \geq 3$. For each $P \in\left\langle\nu_{d}(2 O)\right\rangle \backslash\left\{\nu_{d}(O)\right\}$ let $E_{P} \subseteq\{1, \ldots, m\}$ be a minimal subset such that $P \in\left\langle\cup_{i \in E_{P}}\left\{2 O, L_{i}\right\}\right\rangle$. We have $\rho(P)=\sharp\left(E_{P}\right), r_{T_{m, d}}(P)=d \rho(P)$ and $O \notin S$ for each finite subset $S \subset T_{m}$ with $\sharp(S)=d \rho(P)$ and $P \in\left\langle\nu_{d}(S)\right\rangle$.

Proof. For any $I \subseteq\{1, \ldots, n\}, I \neq \emptyset$, the linear space $\left\langle\nu_{d}(2 O)\right\rangle \cap\left\langle\nu_{d}\left(\cup_{i \in I} L_{i}\right)\right\rangle$ is spanned by the lines $\left\langle\nu_{d}\left(\left\{2 O, L_{i}\right\}\right)\right\rangle, i \in I$ spanned by degree two zerodimensional scheme $\nu_{d}\left(\left\{2 O, L_{i}\right\}\right)$. Therefore the set $E_{P}$ is unique by Lemma 1 and hence the integer $\sharp\left(E_{P}\right)$ is well-defined. Obviously $\sharp\left(E_{P}\right)=\rho(P)$. If $\rho(P)=1$, then Proposition 2 is true by a theorem of Sylvester ([4, Theorem 23], [8, Theorem 5.1]) and concision ([7, Exercise 3.2.2.2]). Hence we may assume $\rho(P) \geq 2$. By Proposition 1 it is sufficient to do the case $\rho(P)=m$. Since $\left\langle\nu_{d}(2 O)\right\rangle$ is the linear span of the lines $\left\langle\nu_{d}\left(\left\{2 O, L_{i}\right\}\right)\right\rangle, i=1, \ldots, n$, and $\rho(P)=m$, there are points $P_{i} \in\left\langle\nu_{d}\left(\left\{2 O, L_{i}\right\}\right)\right\rangle \backslash\left\{\nu_{d}(O)\right\}, i=1, \ldots n$, such that $P \in\left\langle\left\{P_{1}, \ldots, P_{n}\right\}\right\rangle$. For each $P_{i}$ there is $S_{i} \subset L_{i}$ such that $P \in\left\langle S_{i}\right\rangle$ (e.g. by the quoted theorem of Sylvester or by [8, Proposition 4.1]). Hence $r_{T_{m, d}}(P) \leq m d$. Hence it is sufficient to prove the opposite inequality and that no subset of $T_{m}$ evincing $r_{T_{m, d}}(P)$ contains $O$. Set $Z_{i}:=\left\{2 O, L_{i}\right\}$. Since $d \geq 3$, the scheme $Z_{i}$ is the only degree two zero-dimensional subscheme of $\mathbb{P}^{m}$ such that $P_{i} \in\left\langle\nu_{d}\left(Z_{i}\right)\right\rangle$. Fix a set $S^{\prime} \subset T_{m}$ such that $\sharp\left(S^{\prime}\right) \leq m d-1, O \notin S^{\prime}$ and set $S:=\{O\} \cup S^{\prime}$. Increasing if necessary $S^{\prime}$ we may assume $\sharp\left(S^{\prime}\right)=m d-1$.

Assume for the moment $\sharp\left(S^{\prime} \cap L_{i}\right) \leq d$ for all $i=1, \ldots, n$. There is a unique index $j \in\{1, \ldots, n\}$ such that $\sharp\left(S^{\prime} \cap L_{j}\right)=m-1$. Write $T:=\cup_{i \neq j} T_{i}, S_{2}:=$ $S^{\prime} \cap L_{j}$ and $S_{1}:=S \cap T$. We have $O \in S_{1}, S_{1} \cap S_{2}=\emptyset$ and $\left\langle\nu_{d}\left(S_{1}\right)\right\rangle=\left\langle\nu_{d}(T)\right\rangle$. We have $\left\langle\nu_{d}\left(S_{2}\right)\right\rangle \cap\left\langle\nu_{d}\left(Z_{j}\right)\right\rangle=\emptyset$ because $h^{1}\left(L_{j}, \mathcal{I}_{W}(d)\right)=0$ for each zerodimensional scheme $W \subset L_{j}$ with $\operatorname{deg}(W) \leq d+1$. Hence $\left\langle\nu_{d}\left(Z_{j} \cup S_{2}\right)\right\rangle=\nu_{d}\left(L_{j}\right)$ and $\left\langle\nu_{d}\left(S_{2}\right)\right\rangle \cap\left\langle\nu_{d}\left(Z_{j}\right)=\emptyset\right.$. Since $O \in S_{1}$ and the points $P_{j}$ and $\nu_{d}(O)$ span the line $L_{j}$, we get that $P \in\left\langle\nu_{d}(S)\right\rangle$ if and only if $P_{j} \in\left\langle\nu_{d}\left(S_{j}\right)\right\rangle$ for all $j=1,2$. We saw that $\left\langle\nu_{d}\left(S_{2}\right)\right\rangle \cap\left\langle\nu_{d}\left(Z_{2}\right)=\emptyset\right.$ and hence $P_{2} \notin\left\langle\nu_{d}\left(S_{2}\right)\right\rangle$.

Now assume $\sharp\left(S^{\prime} \cap L_{i}\right) \geq d+1$ for some $i$. We still have at least one index $j$ with $\sharp\left(S^{\prime} \cap L_{j}\right) \leq m-1$ and we work in the same way, increasing if necessary $S$ (even with cardinality $>m d$ ) to get $\sharp\left(S \cap L_{i}\right) \geq d$ for all $i \neq j$ and 
$\left\langle\nu_{d}(T)\right\rangle=\left\langle\nu_{d}(T \cap S)\right\rangle$.

Example 2. Assume $m \geq 2$. Let $L \subset \mathbb{P}^{m}$ be a line such that $O \in L$ and $L \neq L_{i}$ for each $i$. Fix any $P \in\left\langle\nu_{d}(\{2 O, L\})\right\rangle \backslash \nu_{d}(O)$. We have $r_{m, d}(P)=d$ ([4, Theorem 32]). By Proposition 2 we have $r_{T_{m, d}}(P)=\rho(P) d$ and hence we may take (for suitable) as $r_{T_{m, d}}(P)$ any integer $x d$ with $x \in\{2, \ldots m\}$. For a general $P \in\left\langle\nu_{d}(2 O)\right\rangle$ we have $r_{m, d}(P)=d, r_{T_{m, d}}(P)=m d$ and hence $r_{T_{m, d}}(P)-r_{m, d}(P)=(m-1) d$.

Proof of Theorem 1. First assume $\rho(P)=0$. Fix any $P_{1}, \ldots, P_{n}$ as in Remark 1. We will take suitable $S_{i} \subset L_{i}$, set $S:=S_{1} \cup \cdots \cup S_{n}$, prove that $P \in\left\langle\nu_{d}(S)\right\rangle$ and give an upper bound for $\sharp(S)$. If $P_{i}$ is not contained in the tangential surface of $\left\langle\nu_{d}\left(L_{i}\right)\right\rangle$, then it has rank $\leq d-1$ with respect to the degree $d$ rational normal curve $\nu_{d}\left(L_{i}\right)$ by Sylvester's theorem. In this case we take as $S_{i}$ any subset of $L_{i}$ evincing this rank. Hence in this case $\sharp\left(S_{i}\right) \leq d-1$. Now assume that $P_{i}$ is contained in the tangential surface of $\left\langle\nu_{d}\left(L_{i}\right)\right\rangle$. In this case $P_{i}$ has rank $d$ with respect to $\nu_{d}\left(L_{i}\right)$, but there is $S_{i} \subset L_{i}$ such that $\sharp\left(S_{i}\right)=d, O \in S_{i}$ and $P_{i} \in\left\langle\nu_{d}\left(S_{i}\right)\right\rangle$ ([1, part (b3) of Proposition 1]). Set $S:=S_{1} \cup \cdots \cup S_{n}$. We have $\sharp(S) \leq m(d-1)+1$ and equality holds only if some $P_{i}$ is in the tangential surface of $\nu_{d}\left(L_{i}\right)$. Since $P \in\left\langle\left\{P_{1}, \ldots, P_{n}\right\}\right\rangle$, we have $P \in\left\langle\nu_{d}(S)\right\rangle$. Now assume $\rho(P)>0$. If $\rho(P)=m$, then use Proposition 2. Assume $0<\rho(P)<m$ and set $E:=\left\{i \in\{1, \ldots, n\}: P_{i} \in\left\langle\left\{2 O, L_{i}\right\}\right\rangle\right\}$. For each $i \in\{1, \ldots, n\} \backslash E$ take $S_{i}$ as above. For each $i \in E$ fix $S_{i} \subset L_{i}$ evincing the rank of $P_{i}$ with respect to $\nu_{d}\left(L_{i}\right)$. In this way we get $r_{T_{m, d}} \leq m(d-1)+1+\rho(P)$. To conclude the proof we only need to check the case $\rho(P)=m-1$. Assume $d \geq 5$. Without losing generality we may assume $E=\{1, \ldots, n-1\}$. If $P_{n}$ has rank $\leq d-1$ with respect to $\nu_{d}\left(L_{n}\right)$, then the proof just given shows that $r_{T_{m, d}}(P)<m d$. Now assume that $P_{i}$ has rank $d$. Therefore there is $Q \in L_{n}$ such that $P_{n} \in\left\langle\nu_{d}\left(\left\{2 Q, L_{n}\right\}\right)\right\rangle$, where $\left\{2 Q, L_{n}\right\}$ denote the degree two effective divisor of $L_{n}$ with $Q$ as its support. Since $n \notin E$, we have $Q \neq O$. Fix $Q_{n} \in\left\langle\left\{P_{n}, \nu_{d}(O)\right\rangle \backslash\left\{P_{n}, \nu_{d}(O)\right\}\right.$. We have $Q_{n} \in\left\langle\left\{\left\{2 Q, L_{n}\right\}, \nu_{d}(O)\right\}\right\rangle$ and hence $Q_{n}$ has border rank $\leq 3$ with respect to $\nu_{d}\left(L_{n}\right)$. Assume the existence of a connected degree 2 zero-dimensional scheme $W \subset L_{n}$ such that $Q_{n} \in\left\langle\nu_{d}(W)\right\rangle$. Call $O^{\prime}$ the support of $W$. Since $d \geq 5$, we have $\nu_{d}(O) \notin\left\langle\left\{2 Q, L_{n}\right\}\right\rangle$. Hence $O^{\prime} \neq Q$. Since $n \notin E$, we have $O^{\prime} \neq O$. Hence $W \cap\{Q, O\}=\emptyset$. Since $d \geq 4$ and $\operatorname{deg}\left(W \cup\{O\} \cup\left\{2 Q, L_{n}\right\}\right) \leq$ 5 , we have $h^{1}\left(L_{n}, \mathcal{I}_{W \cup\{O\} \cup\left\{2 Q, L_{n}\right\}}(d)\right)=0$. Since $W \cap\{Q, O\}=\emptyset$, we get $\operatorname{dim}\left(\left\langle\nu_{d}\left(W \cup\{O\} \cup\left\{2 Q, L_{n}\right\}\right)\right\rangle\right)=4$. Hence $\left\langle\nu_{d}(W)\right\rangle \cap\left\langle\nu_{d}\left(\{O\} \cup\left\{2 Q, L_{n}\right\}\right)\right\rangle=\emptyset$, contradicting the fact that $\left.Q_{n} \in\left\langle\nu_{d}(W)\right\rangle \cap\left\langle\nu_{d}\{O\} \cup\left\{2 Q, L_{n}\right\}\right)\right\rangle$. Hence $Q_{n}$ has border rank $\geq 3$ and so rank $\leq d-1$ ([4, Theorem 23],[8, Theorem 4.1]). Take $A_{n} \subset L_{n}$ such that $\sharp\left(A_{n}\right) \leq d-1$ and $Q_{n} \in\left\langle\nu_{d}\left(A_{n}\right)\right\rangle$. Take $Q_{i} \in\left\langle\nu_{d}(O), P_{i}\right\rangle$, 
$i=1, \ldots, n-1$, such that $P \in\left\langle\left\{Q_{1}, \ldots, Q_{n}\right\}\right.$. Take $A_{i} \subset L_{i}, 1 \leq i \leq n-1$, such that $\sharp\left(A_{i}\right) \leq d$ and $Q_{i} \in\left\langle\nu_{d}\left(A_{i}\right)\right\rangle$ and set $A:=A_{1} \cup \cdots \cup A_{n}$. We have $\sharp(A)<m d$ and $P \in\left\langle\nu_{d}(A)\right\rangle$.

Remark 2. Take $\rho(P)=m-1$ and assume $d \geq 5, E=\{1, \ldots, n-1\}$, $P \in\left\langle\left\{P_{1}, \ldots, P_{n}\right\}\right\rangle$ with $P_{i} \in\left\langle\nu_{d}\left(L_{i}\right)\right\rangle$ and call $b$ (resp. $r^{\prime}$ ) the border rank (resp. the rank) of $P_{n}$ with respect to $P_{n}$. We have $b \leq\lceil(d+1) / 2\rceil$ and either $r^{\prime}=b$ or $r^{\prime}=d+2-b^{\prime}$. We saw in the proof of Theorem 1 that $r_{T_{m, d}}(P) \leq(m-1) d+r^{\prime}$. Now assume $r^{\prime}=d$. We claim that $r_{T_{m, d}}(P) \leq m d-2$. If $m=2$, then the claim is [3, Lemma 5.4]. If $m>2$, then using the result for $m=2$ (say for $L_{n-1} \cup L_{n}$ ) and taking suitable $A_{i} \subset L_{i}, i=1, \ldots, m-2$, we get the inequality $r_{T_{m, d}}(P) \leq m d-2$.

Example 3. Fix $O \in \mathbb{P}^{m}, m \geq 2$. Here we describe all zero-dimensional schemes $Z \subset \mathbb{P}^{m}$ such that $\operatorname{deg}(Z)=m+2, Z \supset 2 O$ and either $Z$ is Gorenstein or $Z$ is in linearly general position.

(a) We first check that these two conditions are equivalent for zerodimensional schemes $Z \subset \mathbb{P}^{m}$ such that $\operatorname{deg}(Z)=m+2, Z \supset 2 O$. Write $\mathbb{P}^{m}=\mathbb{P}(W)$ with $W$ an $(m+1)$-dimensional vector space. First assume that $Z$ is in linearly general position. Since $Z \supset 2 O$ and $m \geq 2, Z$ is not curvilinear. By [5, Theorem 1.3] $Z$ is Gorenstein. Now assume that $Z$ is not in linearly general position, i.e. there is a hyperplane $H \subset \mathbb{P}^{m}$ such that $\operatorname{deg}(H \cap Z) \geq m+1$. Since $2 O \nsubseteq H$, we get $\operatorname{deg}(H \cap Z)=m+1$ and $\operatorname{Res}_{H}(Z)=\{O\}$. Hence for each hyperplane $N \subset \mathbb{P}^{n}$ with $O \in N$ we have $Z \subset H \cup N$. Fix a system of homogeneous coordinates $x_{0}, \ldots, x_{m}$ of $\mathbb{P}^{m}$ such that $O=(1: 0: \ldots: 0)$, $H=\left\{x_{1}=0\right\}$ and set $z_{i}:=x_{i} / x_{0}, i=1, \ldots, n$. We get that $z_{1} z_{j \mid Z} \equiv 0$ for each $j=1, \ldots, m$, i.e. that $z_{1}$ represents an element of the socle of $\mathcal{O}_{Z, O}$ which is not in $\mu^{2}$, where $\mu$ is the maximal ideal of $\mathcal{O}_{Z, O}$. Since $Z \supset 2 O$ and $\operatorname{deg}(Z)=\operatorname{deg}(2 O)+1$, the $\mathbb{C}$-vector space $\mu^{2} / \mu^{3}$ has dimension $1, \mu^{3}=0$ and any element of $\mu^{2}$ is contained in the socle of $\mathcal{O}_{Z, O}$. Hence $\mathcal{O}_{Z, O}$ has socle degree $\geq 2$, i.e. it is not Gorenstein.

(b) Now we take $Z$ in linearly general position and hence Gorenstein. For each hyperplane $H \subset \mathbb{P}^{m}$ we have $\operatorname{deg}(Z \cap H)=m$ and hence $Z \cap H=\{2 O, H\}$ and $\operatorname{deg}\left(\operatorname{Res}_{H}(Z)\right)=2$. Therefore there is a unique line $L$ spanned by the scheme $\operatorname{Res}_{H}(Z)$. We get $Z \subset H \cup N$ for each hyperplane $N \supset L$. From the residual exact sequence

$$
0 \rightarrow \mathcal{I}_{\operatorname{Res}_{H}(Z)}(t-1) \rightarrow \mathcal{I}_{Z}(t) \rightarrow \mathcal{I}_{Z \cap H, H}(2) \rightarrow 0
$$

we get $h^{1}\left(\mathcal{I}_{Z}(t)\right)=0$ for all $t \geq 2$. Hence $h^{0}\left(\mathcal{I}_{Z}(2)\right)=\left(\begin{array}{c}m+2 \\ 2\end{array}\right)-m-2$. For $m=2$ we get that $Z$ is the complete intersection of two singular conics through 
$O$ (and the converse holds).

Proposition 3. Assume $d \geq 3$. Take $Z \subset \mathbb{P}^{m}$ as in Example 3 with $Z_{\text {red }}=\{O\}$ and assume $T_{m} \supset Z$. Take $P \in\left\langle\nu_{d}(Z)\right\rangle$ such that $P \notin\left\langle\nu_{d}\left(Z^{\prime}\right)\right\rangle$ for any $Z^{\prime} \subsetneq Z$. Fix a finite set $B \subset T_{n}$. Then there is $S \subset T_{n} \backslash(\{O\} \cup B)$ such that $\sharp\left(S \cap L_{i}\right)=d-1$ for all $i$ and $P \in\left\langle\nu_{d}(S)\right\rangle$.

Proof. The case $m=2$ is true ([3, Lemma 5.4]) and hence we may assume $m>2$ and use induction on $m$.

We first check that $\rho(P)=0$. Take $\left(P_{1}, \ldots, P_{m}\right)$ with $P_{i} \in\left\langle\nu_{d}\left(L_{i}\right)\right\rangle$ and $P \in\left\langle\left\{P_{1}, \ldots, P_{m}\right\}\right\rangle$. Assume that $P_{i} \in\left\langle\nu_{d}\left(\left\{2 O, L_{i}\right\}\right)\right\rangle$ for some $i$. Fix a homogeneous system of coordinates $x_{0}, \ldots, x_{m}$ such that $O=(1: 0: \cdots: 0)$ and $L_{j}=\left\{x_{j}=0\right\}$. Let $H \subset \mathbb{P}^{m}$ be the hyperplane $\left\{x_{i}=0\right\}$. Since $Z$ is in linearly general position, the scheme $Z \cap H$ is the 2-point $\{2 O, H\}$ of $H$ and it has degree $m$. Hence the residual scheme $\operatorname{Res}_{H}(Z)$ has degree two and it is supported by $O$. We claim that $\operatorname{Res}_{H}(Z)$ is not contained in $H$. Indeed, if it were contained in $H$, then we would get that $x_{i}^{2}$ is in the homogeneous ideal of $Z$. Since all reducible quadrics $\left\{x_{i} x_{j}\right\}, j=1, \ldots, m, j \neq i$, contain $T_{m}$, we would get $x_{j} x_{i \mid Z} \equiv 0$ for each $j=1, \ldots, n$. Hence the local ring $\mathcal{O}_{Z, O}$ would have socle degree $\geq 2$ (its socle also contains a generator as a $\mathbb{C}$-vector space of the ideal sheaf of $2 O$ in $Z$ ), a contradiction. Fix any finite set $A \subset \cup_{j \neq i} L_{j}$ such that $\cup_{j \neq i}\left\{P_{j}\right\} \subset\left\langle\nu_{d}(A)\right\rangle$. We get $P \in\left\langle\nu_{d}\left(\left\{2 O, L_{i}\right\} \cup H\right\rangle\right.$ and hence $P \in\left\langle\nu_{d}(2 H)\right\rangle$. Since the quadric $Q$ contains $2 O, \operatorname{deg}(Z)=\operatorname{deg}(2 O)+1$ and $P \notin\left\langle\nu_{d}(2 O)\right\rangle$, we have $\left\langle\nu_{d}(Z)\right\rangle=\left\langle\{P\} \cup \nu_{d}(2 O)\right\rangle$. Hence $\left\langle\nu_{d}(Z)\right\rangle \subset\left\langle\nu_{d}(2 H)\right\rangle$. Let $g$ be a degree $d-2$ polynomial not vanishing at $O$. The polynomial $g x_{i}^{2}$ vanishes on $2 H$, but it does not vanish on $Z$. Hence $\nu_{d}(Z) \nsubseteq\left\langle\nu_{d}(2 H)\right\rangle$, a contradiction.

Since $\rho(P)=0$, to prove Proposition 3 using the proof of Theorem 1 it is sufficient to prove that no $P_{i}$ has rank $d$ with respect to the rational normal curve $\nu_{d}\left(L_{i}\right)$. Assume that this is the case for at least one index, $i$. Since $\rho(P)=0$, there is a point $Q \in L_{i} \backslash\{O\}$ such that $P_{i} \in\left\langle\nu_{d}\left(\left\{2 Q, L_{i}\right\}\right)\right\rangle$. Since $d \geq 4$, we have $h^{1}\left(L_{i}, \mathcal{I}_{W}(4)\right)=0$ for each zero-dimensional scheme $W \subset L_{i}$ with $\operatorname{deg}(W) \leq 5$. Hence the only point of the plane $\left\langle\nu_{d}\left(\left\{2 Q, L_{i}\right\}\right)\right\rangle$ contained in the linear span of a degree 2 divisors of $\nu_{d}\left(L_{i}\right)$ are the points of the lines $\left\langle\left\{\nu_{d}(Q), \nu_{d}(O)\right\}\right\rangle$. Hence each point $P_{i}^{\prime} \in\left\langle\left\{P_{i}, \nu_{d}(O)\right\}\right\rangle \backslash\left\{P_{i}\right\}$ has rank $\leq d-3$ with respect to $\nu_{d}\left(L_{i}\right)$. However, fixing $P_{i}^{\prime}$ we have to change the other points $P_{j}, j \neq i$. We need to check that we may do the same trick simultaneously for all bad indices $i$ without creating new bad indices. Fix an index $j \neq i$ with $P_{j}$ of rank two and call $Q_{j} \subset L_{j}$ the only point of $L_{j}$ such that $P_{j} \in$ $\left\langle\nu_{d}\left(\left\{2 Q_{j}, L_{j}\right\}\right)\right\rangle$. We saw that $r_{\nu_{d}\left(L_{j}\right.}\left(P_{j}^{\prime}\right)<d$ for any $P_{j}^{\prime} \in\left\langle\left\{P_{j}, \nu_{d}(O)\right\}\right\rangle \backslash\left\{P_{j}\right\}$ and hence we may simultaneously handle all bad indices. Let $F$ be the set of 
all $j \in\{1, \ldots, m\}$ such that $P_{j}$ has rank in the interval $2 \leq x \leq d-3$. Fix any $j \in F$. Since the tangential variety $\tau\left(\nu_{d}\left(L_{j}\right)\right)$ of $\nu_{d}\left(L_{j}\right)$ is closed, we see that moving by a small amount each bad $P_{i}$ the corresponding point $P_{j}^{\prime}$ does not go to $\tau\left(\nu_{d}\left(L_{j}\right)\right)$ and hence $P_{j}^{\prime}$ has rank $\leq d-3$. Now assume the existence of some $j \in\{1, \ldots, m\}$, say $j=n$, with $P_{n}$ of rank 1 , i.e. with $P_{m} \in L_{m}$. Fix $P^{\prime} \in\left\langle T_{m-1, d}\right\rangle$ such that $P \in\left\langle\left\{P^{\prime}, P_{n}\right\}\right\rangle$; apply Proposition 3 to $T_{m-1}$ and then add 1 to get $r_{T_{m, d}}(P) \leq(m-1)(d-1)+1$. Therefore for a general choice of $\left(P_{1}^{\prime}, \ldots, P_{m}^{\prime}\right)$ with $P_{i}^{\prime} \in\left\langle\nu_{d}\left(L_{i}\right)\right\rangle$ and $P \in\left\langle\left\{P_{1}^{\prime}, \ldots, P_{m}^{\prime}\right\}\right\rangle$ each $P_{i}^{\prime}$ has rank $\leq d-3$ with respect to $\nu_{d}\left(L_{i}\right)$.

The additional condition imposed by the set $B$ in the statement of Proposition 3 is related to the definition of open rank ([6]).

In the case $m=3$ we can prove the inequality opposite to Proposition 3 and hence that $r_{T_{3, d}}(P)=r_{m, d}(P)=3 d-3$.

Proposition 4. Take $Z$ as in Example 3 and assume $m=3$ and that $Z \subset T_{3}$. Fix an integer $d \geq 7$ and $P \in\left\langle\nu_{d}(Z)\right\rangle$ such that $P \notin\left\langle\nu_{d}\left(Z^{\prime}\right)\right\rangle$ for all $Z^{\prime} \subsetneq Z$. Then $r_{3, d}(P) \geq 3 d-3$ and there is no set $S \subset \mathbb{P}^{3}$ with $\sharp(S)=3 d-3$ and $O \in S$.

Proof. In order to obtain a contradiction we assume the existence of a set $B \subset \mathbb{P}^{3}$ such that $\sharp(B \cup\{O\}) \leq 3 d-3$ and $P \in\left\langle\nu_{d}(B)\right\rangle$. Taking $B$ minimal we may also assume $P \notin\left\langle\nu_{d}\left(B^{\prime}\right)\right\rangle$ for all $B^{\prime} \subsetneq B$. Set $W_{0}:=\operatorname{deg}(Z \cup S)$. Notice that for each line $L \subset \mathbb{P}^{3}$ either $O \notin L$ and $\operatorname{deg}(L \cap Z)=0$ or $O \in L$ and $\operatorname{deg}(Z \cap L)=2$. Hence either $\operatorname{deg}\left(L \cap W_{0}\right)=\sharp(L \cap B)$ (case $O \notin L$ ) or $\operatorname{deg}\left(W_{0}\right)=2+\sharp(B \cap(L \backslash\{O\}))$ (case $\left.O \in L\right)$. By assumption $w_{0}:=\operatorname{deg}\left(W_{0}\right) \leq$ $3 d+1$. Let $H_{1} \subset \mathbb{P}^{3}$ be a plane such that $e_{1}:=\operatorname{deg}\left(W_{0} \cap H_{1}\right)$ is maximal. Set $W_{1}:=\operatorname{Res}_{H_{1}}\left(W_{0}\right)$. Fix an integer $i \geq 2$ and assume to have defined the integers $e_{j}$, the hyperplanes $H_{j}$ and the schemes $W_{j}$ for all $j=1, \ldots, i-1$. Let $H_{i}$ be any plane such that $e_{i}:=\operatorname{deg}\left(H_{i} \cap H_{i-1}\right)$ is maximal. Set $W_{i}:=\operatorname{Res}_{H_{i}}\left(W_{i-1}\right)$. We have $e_{i} \geq e_{i+1}$ for all $i$. For each integer $i>0$ we have an exact sequence

$$
0 \rightarrow \mathcal{I}_{W_{i}}(d-i) \rightarrow \mathcal{I}_{W_{i-1}}(d+1-i) \rightarrow \mathcal{I}_{W_{i-1} \cap H_{i}, H_{i}}(d+1-i) \rightarrow 0
$$

Since $h^{1}\left(\mathcal{I}_{W_{0}}(d)\right)>0([2$, Lemma 1$])$, the exact sequence $(2)$ show the existence of an integer $i>0$ such $h^{1}\left(H_{i}, \mathcal{I}_{W_{i-1} \cap H_{i}, H_{i}}(d+1-i)\right)>0$. We call $g$ the minimal such an integer. Since any degree 3 zero-dimensional subscheme of $\mathbb{P}^{3}$ is contained in a plane, if $e_{i} \leq 2$, then $W_{i}=\emptyset$ and $e_{j}=0$ for all $j>i$. Since $w_{0} \leq 3 d+1$, we get $e_{d+1}=0$ and $e_{d} \leq 1$. Since $h^{1}\left(\mathcal{O}_{\mathbb{P}^{3}}(d)\right)=h^{1}\left(\mathcal{I}_{Q}\right)=0$ for any $Q \in \mathbb{P}^{3}$, we get $g \leq d$. By [4, Lemma 34] either $e_{g} \geq 2(d+1-g)+2$ or there is a line $L \subset H_{g}$ such that $\operatorname{deg}\left(L \cap W_{i-1}\right) \geq d+3-g$. Assume for the 
moment $g \geq 2$ and $e_{g} \leq 2(d+1-g)+1$. Since $e_{g}>0, W_{i-2}$ spans $\mathbb{P}^{3}$. Hence $e_{g-1} \geq d+4-g$ in all cases. Hence if $g \geq 2$ we get $3 d+1 \geq g(d+4-g)-1$. Hence $1 \leq g \leq 2$.

(a) Assume $g=2$. Since $e_{2} \leq(3 d+1) / 2 \geq 2(d-1)+1$, there is a line $L \subset H_{2}$ such that $\operatorname{deg}\left(L \cap W_{1}\right) \geq d+1$. Let $N_{1} \subset \mathbb{P}^{3}$ be a plane containing $L$ and such that $f_{1}:=\operatorname{deg}\left(W_{0} \cap L\right)$ is maximal among the planes containing $L$. Since $Z$ spans $\mathbb{P}^{3}$, we have $f_{1} \geq d+2$. Set $Z_{0}:=W_{0}$ and $Z_{1}:=\operatorname{Res}_{N_{1}}\left(Z_{0}\right)$. Let $N_{2}$ be a plane such that $f_{2}:=\operatorname{deg}\left(N_{2} \cap Z_{1}\right)$. Set $Z_{1}:=\operatorname{Res}_{N_{2}}\left(Z_{1}\right)$. Define inductively the plane $N_{i}, i \geq 3$, the scheme $Z_{i}:=\operatorname{Res}_{N_{i}}\left(Z_{i-1}\right)$ and the integer $f_{i}:=$ $\operatorname{deg}\left(N_{i} \cap Z_{i-1}\right)$ with $f_{i}$ maximal among all planes. We have $f_{i} \geq f_{i+1}$ for all $i \geq 3$ and if $f_{i} \leq 2$, then $f_{i+1}=0$ and $Z_{i}=\emptyset$. We have the residual exact sequence similar to (2) with $N_{i}$ instead of $H_{i}, Z_{i-1}$ instead of $W_{i-1}$ and $Z_{i}$ instead of $W_{i}$. Hence there is an integer $i>0$ such that $h^{1}\left(N_{i}, \mathcal{I}_{N_{i} \cap Z_{i-1}, H_{i}}(d+1-i)\right)>0$ and we call $g^{\prime}$ the first such an integer. Since $\sum_{i \geq 2} f_{i} \leq 2 d-1$ and $f_{i+1}=0$ if $f_{i} \leq 2$, we have $g^{\prime}<d$. Hence either $f_{g^{\prime}} \geq 2\left(d+1-g^{\prime}\right)+2$ or there is a line $R \subset N_{g^{\prime}}$ such that $\operatorname{deg}\left(R \cap Z_{i-1}\right) \geq d+3-g^{\prime}$. Assume for the moment $g^{\prime} \geq 3$. We get $f_{g^{\prime}-1} \geq d+4-g^{\prime}$. Hence $2 d-1 \geq \sum_{i \geq 2} f_{i} \geq\left(g^{\prime}-1\right)\left(d+4-g^{\prime}\right)-1$. We get $1 \leq g^{\prime} \leq 2$.

(a1) Assume $g^{\prime}=2$. Since $f_{1} \geq d+2$, we have $f_{2} \leq 2(d-1)+1$. By $[4$, Lemma 34] there is a line $R \subset N_{2}$ such that $\operatorname{deg}\left(R \cap Z_{1}\right) \geq d+1$.

(a1.1) Assume $L \cap R=\emptyset$. Since $Z$ is connected and spans $\mathbb{P}^{3}$, there is a smooth quadric $Q \subset \mathbb{P}^{3}$ containing $L \cup R$ and with $\operatorname{deg}\left(Q \cap W_{0}\right) \geq 1+\operatorname{deg}\left(W_{0} \cap\right.$ $R)+\operatorname{deg}\left(W_{0} \cap L\right) \geq 2 d+3$. Since $\operatorname{Res}_{Q}\left(W_{0}\right)$ has degree $\leq d-2$, we have $h^{1}\left(\mathcal{I}_{\operatorname{Res}_{Q}\left(W_{0}\right)}(d-2)\right)=0$. Since $Z$ is connected, [3, Lemma 5.1] gives $W_{0} \subset Q$. Since $Q$ is a smooth quadric, we have $Q \nsupseteq Z$, a contradiction.

(a1.2) Assume $L \cup R \neq \emptyset$. Since $\operatorname{deg}(R \cap(B \backslash B \cap L)) \geq d+1-\operatorname{deg}(R \cap Z)=$ $d-1>0$, we have $L \neq R$. Hence $L \cap R$ is a point, $O^{\prime}$ and $\Pi:=\langle L \cup R\rangle$ is a plane. First assume $O^{\prime} \neq O$. We get $W_{0} \cap(L \cup R) \geq 2 d+2$, because $B \cap W_{1} \subseteq B \backslash B \cap L$. Hence $\operatorname{deg}\left(\operatorname{Res}_{\Pi}\left(W_{0}\right)\right) \leq d-1$. Therefore $h^{1}\left(\mathcal{I}_{\operatorname{Res}_{\Pi}\left(W_{0}\right)}(d-1)\right)=0$. Since $Z$ is connected, [3, Lemma 5.1] gives $Z \cup B \subset \Pi$, contradicting the inclusion $Z \supset 2 O$. Now assume $O^{\prime}=O$. We may also assume $h^{1}\left(\mathcal{I}_{\operatorname{Res}_{\Pi}\left(W_{0}\right)}(d-1)\right)>0$. In this case we have $\operatorname{deg}\left(\operatorname{Res}_{\Pi}\left(W_{0}\right)\right) \geq 3 d+1-(2 d-1)$. By [4, Lemma 34] there is a line $D \subset \mathbb{P}^{3}$ such that $\operatorname{deg}\left(D \cap \operatorname{Res}_{\Pi}\left(W_{0}\right)\right) \geq d+1$. Looking at $\sharp(B \backslash B \cap R)$ and $\sharp(B \backslash B \cap L)$ we see that $D \neq R$ and $D \neq L$. Since $e_{1} \leq 3 d+1-d-1$, we have $D \nsubseteq \Pi$. First assume $O \in D \cap L \cap R$. We have $h^{0}\left(\mathcal{I}_{D \cup L \cup R}(2)\right)=3$ and a general $Q \in\left|\mathcal{I}_{D \cup L \cup R}(2)\right|$ is an irreducible quadric cone. Since $\operatorname{deg}_{Q}\left(\operatorname{Res}_{Q}\left(W_{0}\right)\right) \leq d-1$, we have $h^{1}\left(\mathcal{I}_{\operatorname{Res}_{Q}\left(W_{0}\right)}(d-2)\right)=0$. By [3, Lemma 5.1] we get $W_{0} \subset Q$. Since $\operatorname{deg}(L \cap Z)=\operatorname{deg}(D \cap Z)=\operatorname{deg}(R \cap Z)=2$, we have $\sharp(B \cap(D \backslash\{O\})) \geq d-1$, $\sharp(B \cap(R \backslash\{O\})) \geq d-1$ and $\sharp(B \cap(L \backslash\{O\}) \geq d-1$ and hence $\sharp(B \cup\{O\}) \geq 3 d-2$, 
a contradiction. If either $D \cap L=\emptyset$ or $D \cap R=\emptyset$, then we conclude as in step (a1.1). If $D$ intersects exactly one of the line $R$ and $L$. In this case $R \cup L \cup D$ is contained in a smooth quadric and we get a contradiction as in step (a1.1).

(a2) Assume $g^{\prime}=1$. Since $h^{1}\left(N_{1}, \mathcal{I}_{W_{0} \cap N_{1}}(d)\right)>0$ and $f_{1} \leq e_{1} \leq 2 d$, there is a line $J \subset N_{1}$ such that $\operatorname{deg}\left(J \cap W_{0}\right) \geq d+2$. Since $L \subset N_{1}$ and $\operatorname{deg}\left(L \cap W_{1}\right) \geq d+1$, we get $J=L$. We work as in step (b).

(b) Assume $g=1$. Since $h^{1}\left(H_{1}, \mathcal{I}_{W_{0} \cap H_{1}}(d)\right)>0$, we have $e_{1} \geq d+2$ and hence $\operatorname{deg}\left(W_{1}\right) \leq 2 d-1$. First assume $h^{1}\left(\mathcal{I}_{W_{1}}(d-1)\right)=0$. Since $Z$ is connected, [3, Lemma 5.1] gives $Z \subset H_{1}$, a contradiction. Now assume $h^{1}\left(\mathcal{I}_{W_{1}}(d-1)\right)>0$. Since $\operatorname{deg}\left(W_{1}\right) \leq 2(d-1)$, [4, Lemma 34] gives the existens of a line $L \subset \mathbb{P}^{3}$ such that $\operatorname{deg}\left(L \cap W_{1}\right) \geq d+1$. Fix any plane $M \supset L$. We have $\operatorname{Res}_{H_{1} \cup M}\left(W_{0}\right)=\operatorname{Res}_{M}\left(W_{1}\right)$. Hence $\operatorname{deg}\left(\operatorname{Res}_{M}\left(W_{1}\right)\right) \leq$ $d-2$. Since $\operatorname{deg}\left(\operatorname{Res}_{M}\left(W_{1}\right)\right) \leq 3 d+1-(d+2)-(d+1)=d-2$, we have $h^{1}\left(\mathcal{I}_{\operatorname{Res}_{M}\left(W_{1}\right)}(d-2)\right)=0$. By [2, Lemma 5.1] we get $W_{0} \subset H_{1} \cup M$ and in particular $B \subset H_{1} \cup L$. Set $Z_{0}:=W_{0}$. Let $N_{1}$ be a plane containing $L$ and such that $f_{1}:=\operatorname{deg}\left(N_{1} \cap Z_{0}\right)$ is maximal among the planes containing $L$. Since $Z$ spans $\mathbb{P}^{3}$, we have $f_{1} \geq d+2$. Set $Z_{0}:=W_{0}$ and $Z_{1}:=\operatorname{Res}_{N_{1}}\left(Z_{0}\right)$. Let $N_{2}$ be a plane such that $f_{2}:=\operatorname{deg}\left(N_{2} \cap Z_{1}\right)$. Set $Z_{1}:=\operatorname{Res}_{N_{2}}\left(Z_{1}\right)$. Define inductively the plane $N_{i}, i \geq 3$, the schemes $Z_{i}:=\operatorname{Res}_{N_{i}}\left(Z_{i-1}\right)$ and the integer $f_{i}:=$ $\operatorname{deg}\left(N_{i} \cap Z_{i-1}\right)$ with $f_{i}$ maximal among all planes. We have $f_{i} \geq f_{i+1}$ for all $i \geq 3$ and if $f_{i} \leq 2$, then $f_{i+1}=0$ and $Z_{i}=\emptyset$. We have the residual exact sequence similar to (2) with $N_{i}$ instead of $H_{i}, Z_{i-1}$ instead of $W_{i-1}$ and $Z_{I}$ instead of $W_{i}$. Hence there is an integer $i>0$ such that $h^{1}\left(N_{i}, \mathcal{I}_{N_{i} \cap Z_{i-1}, H_{i}}(d+1-i)\right)>0$ and we call $g^{\prime}$ the first such an integer. As in step (a) we get $g^{\prime} \in\{1,2\}$.

(b1) Assume $g^{\prime}=2$. As in step (a1) we get a line $R \subset N_{2}$ such that $\operatorname{deg}\left(R \cap Z_{1}\right) \geq d+1$. Steps (a1.1) and (a1.2) work verbatim.

(b2) Assume $g^{\prime}=1$. Since $h^{1}\left(N_{1}, \mathcal{I}_{W_{0} \cap N_{1}}(d)\right)>0$ and $f_{1} \leq e_{1} \leq 2 d$, there is a line $J \subset N_{1}$ such that $\operatorname{deg}\left(J \cap W_{0}\right) \geq d+2$. Since $Z$ spans $\mathbb{P}^{3}$, then $f_{1} \geq 1+\operatorname{deg}\left(J \cap W_{0}\right)=d+3$. Since $L \subset N_{1}$ and $\operatorname{deg}\left(L \cap W_{1}\right) \geq d+1$, we get $J=L$. If $h^{1}\left(\mathcal{I}_{Z_{1}}(d-1)\right)=0$, then [3, Lemma 5.1] gives $Z \subset N_{1}$, a contradiction. Now assume $h^{1}\left(\mathcal{I}_{Z_{1}}(d-1)\right)>0$. Since $f_{1} \geq d+3$, we have $\operatorname{deg}\left(Z_{1}\right) \leq 2 d-2$. By [4, Lemma 34] there is a line $R \subset \mathbb{P}^{3}$ such that $\operatorname{deg}\left(R \cap Z_{1}\right) \geq d+1$. Since $B \cap W_{1} \subseteq(B \backslash(\{O\} \cup L)$ we get $R \neq L$. We work as in steps (a1.1) and (a1.2) (here the inequalities are stronger by 1 , because $\operatorname{deg}\left(L \cap Z_{0}\right) \geq d+2$ ).

\section{Acknowledgements}

The author was partially supported by MIUR and GNSAGA of INdAM (Italy). 


\section{References}

[1] E. Ballico, Subsets of the variety $X \subset \mathbb{P}^{n}$ evincing the $X$-rank of a point of $\mathbb{P}^{n}$, Houston J. Math. (to appear).

[2] E. Ballico and A. Bernardi, Decomposition of homogeneous polynomials with low rank, Math. Z. 271 (2012), 1141-1149; DOI 10.1007/s00209-0110907-6.

[3] E. Ballico andA. Bernardi, Stratification of the fourth secant variety of Veronese variety via the symmetric rank, Adv. Pure Appl. Math. 4, no. 2, 215-250 (2013) DOI: 10.1515/apam-2013-0015

[4] A. Bernardi, A. Gimigliano, M. Idà, Computing symmetric rank for symmetric tensors, J. Symbolic. Comput. 46 (2011), no. 1, 34-53.

[5] D. Eisenbud and J. Harris, Finite projective schemes in linearly general position, J. Algebraic Geom. 1 (1992), no. 1, 15-30.

[6] J. Jelisiejew, An upper bound for the Waring rank of a form, arXiv:1305.6957.

[7] J. M. Landsberg, Tensors: Geometry and Applications, Graduate Studies in Mathematics, Vol. 128, Amer. Math. Soc. Providence, 2012.

[8] J. M. Landsberg and Z. Teitler, On the ranks and border ranks of symmetric tensors, Found. Comput. Math. 10 (2010), no. 3, 339-366. 
